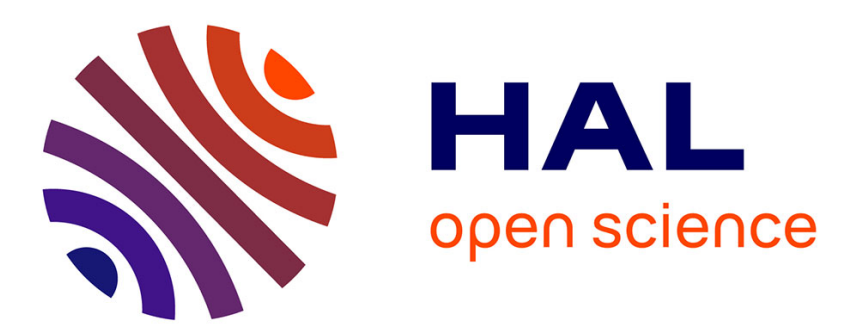

\title{
On a limit of perturbed conservation laws with diffusion and non-positive dispersion
}

\author{
Nabil Bedjaoui, Joaquim M.C. Correia, Youcef Mammeri
}

\section{To cite this version:}

Nabil Bedjaoui, Joaquim M.C. Correia, Youcef Mammeri. On a limit of perturbed conservation laws with diffusion and non-positive dispersion. Communications in Mathematical Sciences, 2016, 10.4310/CMS.2016.v14.n6.a2 . hal-01191885

\section{HAL Id: hal-01191885 \\ https://hal.science/hal-01191885}

Submitted on 2 Sep 2015

HAL is a multi-disciplinary open access archive for the deposit and dissemination of scientific research documents, whether they are published or not. The documents may come from teaching and research institutions in France or abroad, or from public or private research centers.
L'archive ouverte pluridisciplinaire HAL, est destinée au dépôt et à la diffusion de documents scientifiques de niveau recherche, publiés ou non, émanant des établissements d'enseignement et de recherche français ou étrangers, des laboratoires publics ou privés. 


\title{
On a limit of perturbed conservation laws with diffusion and non-positive dispersion
}

\author{
N. Bedjaoui*, J. M.C. Correia ${ }^{+}$, Y. Mammeri* \\ *Laboratoire Amiénois de Mathématique Fondamentale et Appliquée, \\ CNRS UMR 7352, Université de Picardie Jules Verne, France \\ 80069 Amiens, France. \\ + DMat, ECT \& CIMA, IIFA, Universidade de Évora, Évora \\ and CAMGSD, IST, Lisbon, Portugal \\ Email: nabil.bedjaoui@u-picardie.fr,jmcorreia@uevora.pt, youcef.mammeri@u-picardie.fr \\ Corresponding author: nabil.bedjaoui@u-picardie.fr
}

\begin{abstract}
We consider a conservation law perturbed by a linear diffusion and non-positive dispersion $u_{t}+f(u)_{x}=\varepsilon u_{x x}-\delta\left(\left|u_{x x}\right|^{n}\right)_{x}$. We prove the convergence of the previous solution to the entropy weak solution of the hyperbolic conservation law $u_{t}+f(u)_{x}=0$, in both cases $n=1$ and $n=2$.
\end{abstract}

Keywords. diffusion, dispersion, KdV equation, Burgers equation, hyperbolic conservation laws, entropy measure-valued solutions.

MS Codes. 35G25,35L65,35Q53,76B15.

\section{Introduction}

We consider the initial value problem

$$
\begin{aligned}
& u_{t}+f(u)_{x}=\varepsilon u_{x x}+\delta g\left(u_{x x}\right)_{x} \\
& u(x, 0)=u_{0}(x),
\end{aligned}
$$

where $\varepsilon$ and $\delta$ are small parameters, and $g$ is a non positive function, and we focus on the specific form

$$
g(v)=-|v|^{n},
$$

where $n \geq 1$. Note that if $n=1, g$ is lipschitz, if $1<n<2, g$ is $C^{1}$ and for $n \geq 2$, g is $C^{2}$.

When $\delta=0$ we reduce to the viscous (generalized) Burgers equation and the approximate solutions $u^{\varepsilon, 0}$ converge to the entropy solution of the hyperbolic equation (called the vanishing viscosity method, see, e.g., Whitham [21] or Kružkov [9])

$$
\begin{aligned}
& u_{t}+f(u)_{x}=0 \\
& u(x, 0)=u_{0}(x) .
\end{aligned}
$$

On the other hand, when $\varepsilon=0$, if we consider the flux function $f(u)=u^{2}$ and the linear dispersion $\delta u_{x x x}$ we obtain the Korteweg-de Vries equation. The approximate solutions $u^{0, \delta}$ do not converge in a strong topology (see Lax-Levermore [11]). So, as parameters $\varepsilon$ and $\delta$ vanish, we are concerned with singular limits and to ensure convergence it is necessary to be in the dominant dissipation regime. 
The pioneer study of these singular limits was given by Schonbek [16] about the (generalized) Korteweg-de Vries-Burgers equation

$$
u_{t}+f(u)_{x}=\varepsilon u_{x x}-\delta u_{x x x} .
$$

In the case of a convex flux function $f(u)$, she proved the convergence of the solutions of this perturbed equation to the entropy solution of (1.3), when both $\varepsilon$ and $\delta$ tend to zero, at least under the condition $\delta=O\left(\varepsilon^{3}\right)$ (depending on the behavior of the flux $f$ ). Also, according to PerthameRyzhic [15], the sharp condition should be $\delta=o(\varepsilon)$. LeFloch-Natalini [13] proved the convergence in the case of a nonlinear viscosity function $\beta$ and linear capillarity

$$
u_{t}+f(u)_{x}=\varepsilon \beta\left(u_{x}\right)_{x}-\delta u_{x x x} .
$$

Then, Correia-LeFloch [5] improved the estimates in Schonbek [16] and LeFloch-Natalini [13] and for the first time treated the multidimensional equation, but still in the case of a nonlinear viscosity function and linear capillarity. In fact, the dominant dissipation regime is also assured by the nonlinear viscosity. In our case, we consider the reverse situation.

In general for $\varepsilon=0$, like for the Korteweg-de Vries equation, the divergent behaviour is expected, as we are considering "pure-dispersive equations". But, Brenier-Levy [3] considered the fully nonlinear equation

$$
u_{t}+f(u)_{x}=-\delta\left(u_{x x}^{2}\right)_{x}
$$

as a nonlinear generalization of the Korteweg-de Vries equation. Such nonlinear dispersion significantly affects the dispersive behaviour of the solutions that differs completely from the linear case. In particular, Brenier and Levy [3] conjectured that for strictly convex flux functions $f$ and for the following perturbed problem

$$
u_{t}+f(u)_{x}=-\delta g\left(u_{x x}\right)_{x}-\varepsilon u_{x x x x},
$$

we have convergence when $\varepsilon$ and $\delta$ tend to zero under the condition $\varepsilon=o(\delta)$.

The paper is organized as follows. In Section 2, we present the main results of convergence. Section 3 deals with the uniform estimates needed for convergence. Finally, Section 4 is devoted to proving the convergence to the entropy solution of the hyperbolic equation, when both $\varepsilon$ and $\delta$ go to zero.

\section{Main Results}

Two main convergence results are presented. The first one is concerned with $g(v)=-|v|$ (i.e. $n=1$ ) while the second one is devoted to the case $g(v)=-v^{2}$ (i.e. $n=2$ ).

\subsection{Case $f$ convex, $\delta>0$ and $g\left(u_{x x}\right)=-\left|u_{x x}\right|$}

In this case, we prove the following result.

Theorem 2.1 Let $\varepsilon>0, \delta=o\left(\varepsilon^{2}\right)$, and $f: \mathbb{R} \rightarrow \mathbb{R}$ be a convex flux function satisfying

$$
f^{\prime \prime}(u) \leq C\left(1+|u|^{\beta}\right) \text {, where } 0 \leq \beta<3 .
$$

Then, setting $u=u_{\varepsilon, \delta}$ the solution of $(1.1)-(1.2)$, the family solutions $\left(u_{\varepsilon, \delta}\right)$ converges to the entropy solution of (3) - (4).

In the case of the linear dispersion, i.e. $g\left(u_{x x}\right)=u_{x x}$ treated in [16], Schonbek gets for a general flux satisfying $f^{\prime \prime}(u) \leq C$, a convergence with rate $\delta=O\left(\varepsilon^{3}\right)$. Also, when $f^{\prime \prime}(u) \leq C(1+|u|)$, the author obtain the convergence with the rate $\delta=O\left(\varepsilon^{4}\right)$. The case of $g\left(u_{x x}\right)=-\left|u_{x x}\right|$ seems giving a weakly dispersive effects than a classical linear dispersion. 


\subsection{Case $f$ convex, $\delta>0$ and $g\left(u_{x x}\right)=-u_{x x}^{2}$}

Here function $g$ is regular, and we obtain:

Theorem 2.2 Let $\varepsilon>0, \delta=o\left(\varepsilon^{5 / 2}\right)$, and $f: \mathbb{R} \rightarrow \mathbb{R}$ be a convex flux function satisfying

$$
f^{\prime \prime}(u) \leq C\left(1+|u|^{\beta}\right) \text {, where } 0 \leq \beta<1 / 2 .
$$

Then, setting $u=u_{\varepsilon, \delta}$ the solution of $(1.1)-(1.2)$, the family solutions $\left(u_{\varepsilon, \delta}\right)$ converges to the entropy solution of (3) - (4).

The dispersion here is strongly nonlinear but regular, which provides the well-posedness $[1,2]$. We can see that comparing to the results in [16], the rate are quite similar when the flux is convex and satisfies $f^{\prime \prime}(u) \leq C\left(\delta=o\left(\varepsilon^{5 / 2}\right)\right.$ whereas $\delta=O\left(\varepsilon^{3}\right)$ in [16]).

\section{A priori estimates}

Assume that $\eta$ is a regular function and $\varphi$ a function defined by $\varphi^{\prime}=\eta^{\prime} f^{\prime}$, and let us multiply (1.1) by $\eta^{\prime}(u)$. We obtain

$$
\begin{aligned}
\eta(u)_{t}+\varphi(u)_{x}= & \varepsilon\left(\eta^{\prime}(u) u_{x}\right)_{x}-\varepsilon \eta^{\prime \prime}(u) u_{x}^{2} \\
& +\delta\left(\eta^{\prime}(u) g\left(u_{x x}\right)\right)_{x}-\delta \eta^{\prime \prime}(u) u_{x} g\left(u_{x x}\right) .
\end{aligned}
$$

Integrating over $(0, t) \times \mathbb{R}$ with $\eta(u)=|u|^{\alpha+1}$, the conservative terms vanish and we obtain the following lemma.

Lemma 3.1 Let $\alpha \geq 1$ and $g: \mathbb{R} \rightarrow \mathbb{R}$ be any dispersion function. Each solution of (1.1) satisfies for $t \in[0, T]$

$$
\begin{aligned}
\int_{\mathbb{R}}|u(t)|^{\alpha+1} d x & +(\alpha+1) \alpha \varepsilon \int_{0}^{t} \int_{\mathbb{R}}|u|^{\alpha-1} u_{x}^{2} d x d s \\
& +(\alpha+1) \alpha \delta \int_{0}^{t} \int_{\mathbb{R}}|u|^{\alpha-1} u_{x} g\left(u_{x x}\right) d x d s \\
& =\int_{\mathbb{R}}\left|u_{0}\right|^{\alpha+1} d x .
\end{aligned}
$$

Usually, taking $\alpha=1$ in (3.2), we deduce the a priori $L^{2}$ first energy estimates.

Let us introduce the functions $\mathcal{G}$, and $G$ defined by $\mathcal{G}^{\prime \prime}=G^{\prime}=g$, i.e,

$$
G(u)=-\frac{1}{n+1}|u|^{n} u, \text { and } \mathcal{G}=-\frac{1}{(n+1)(n+2)}|u|^{n+2} .
$$

Using the multiplier $(q+2)\left(\left|u_{x}\right|{ }^{q} u_{x}\right)_{x}$ to (1.1), we have

$$
\begin{aligned}
\left((q+2) u_{t}\left|u_{x}\right|^{q} u_{x}\right)_{x}-\left(\left|u_{x}\right|^{q+2}\right)_{t}= & -(q+2)(q+1)\left|u_{x}\right|^{q} u_{x x} f^{\prime}(u) u_{x} \\
& +\varepsilon(q+2)(q+1)\left|u_{x}\right|^{q} u_{x x}^{2} \\
& +\delta(q+2)(q+1)\left|u_{x}\right|^{q} u_{x x} g^{\prime}\left(u_{x x}\right) u_{x x x} \\
= & -(q+1)\left(\left|u_{x}\right|^{q+2}\right)_{x} f^{\prime}(u) \\
& +\varepsilon(q+2)(q+1)\left|u_{x}\right|^{q} u_{x x}^{2} \\
& +\delta(q+2)(q+1) n\left|u_{x}\right|^{q} G\left(u_{x x}\right)_{x},
\end{aligned}
$$


and we get the estimate

$$
\begin{aligned}
\left((q+2) u_{t}\left|u_{x}\right|^{q} u_{x}\right)_{x}-\left(\left|u_{x}\right|^{q+2}\right)_{t}= & -\left((q+1)\left|u_{x}\right|^{q+2} f^{\prime}(u)\right)_{x} \\
& +(q+1)\left|u_{x}\right|^{q+2} u_{x} f^{\prime \prime}(u) \\
& +\varepsilon(q+2)(q+1)\left|u_{x}\right|^{q} u_{x x}^{2} \\
& +\left(\delta(q+2)(q+1) n\left|u_{x}\right|^{q} G\left(u_{x x}\right)\right)_{x} \\
& -\delta(q+2)(q+1) q(n+2) n\left|u_{x}\right|^{q-2} u_{x} \mathcal{G}\left(u_{x x}\right) .
\end{aligned}
$$

Similarly, using the multiplier $(q+2)\left(u_{x}^{q+1}\right)_{x}$ to $(1.1)$, we can write

$$
\begin{aligned}
\left((q+2) u_{t} u_{x}^{q+1}\right)_{x}-\left(u_{x}^{q+2}\right)_{t}= & -(q+2)(q+1) u_{x}^{q+1} f^{\prime}(u) u_{x x} \\
& +\varepsilon(q+2)(q+1) u_{x}^{q} u_{x x}^{2} \\
& +\left(\delta(q+2)(q+1) n u_{x}^{q} G\left(u_{x x}\right)\right)_{x} \\
& -\delta(q+2)(q+1) q(n+2) n u_{x}^{q-1} \mathcal{G}\left(u_{x x}\right),
\end{aligned}
$$

thus

$$
\begin{aligned}
\left((q+2) u_{t} u_{x}^{q+1}\right)_{x}-\left(u_{x}^{q+2}\right)_{t}= & -\left((q+1) u_{x}^{q+2} f^{\prime}(u)\right)_{x} \\
& +(q+1) u_{x}^{q+3} f^{\prime \prime}(u) \\
& +\varepsilon(q+2)(q+1) u_{x}^{q} u_{x x}^{2} \\
& +\left(\delta(q+2)(q+1) n u_{x}^{q} G\left(u_{x x}\right)\right)_{x} \\
& -\delta(q+2)(q+1) q(n+2) n u_{x}^{q-1} \mathcal{G}\left(u_{x x}\right) .
\end{aligned}
$$

Integrating (3.3) and (3.4) over $\mathbb{R} \times[0, t]$ provides

$$
\begin{gathered}
\int_{\mathbb{R}}\left|u_{x}(t)\right|^{q+2} d x+\varepsilon(q+2)(q+1) \int_{0}^{t} \int_{\mathbb{R}}\left|u_{x}\right|^{q} u_{x x}^{2} d x d s \\
=\int_{\mathbb{R}}\left|u_{0}^{\prime}\right|^{q+2} d x-(q+1) \int_{0}^{t} \int_{\mathbb{R}} u_{x}\left|u_{x}\right|^{q+2} f^{\prime \prime}(u) d x d s \\
+\delta(q+2)(q+1) q(n+2) n \int_{0}^{t} \int_{\mathbb{R}} u_{x}\left|u_{x}\right|^{q-2} \mathcal{G}\left(u_{x x}\right) d x d s, \\
\int_{\mathbb{R}} u_{x}(t)^{q+2} d x+\varepsilon(q+2)(q+1) \int_{0}^{t} \int_{\mathbb{R}} u_{x}^{q} u_{x x}^{2} d x d s \\
=\int_{\mathbb{R}}\left(u_{0}^{\prime}\right)^{q+2} d x-(q+1) \int_{0}^{t} \int_{\mathbb{R}} u_{x}^{q+3} f^{\prime \prime}(u) d x d s \\
+\delta(q+2)(q+1) q(n+2) n \int_{0}^{t} \int_{\mathbb{R}} u_{x}^{q-1} \mathcal{G}\left(u_{x x}\right) d x d s .
\end{gathered}
$$

We define now the sets, for $t \in[0, T]$

$$
\mathcal{U}_{t}^{+}=\left\{x \in \mathbb{R} ; u_{x}(x, t)>0\right\},
$$

and

$$
\mathcal{U}_{t}^{-}=\left\{x \in \mathbb{R} ; u_{x}(x, t)<0\right\} .
$$

Adding (3.6) to (3.5) for $q$ odd, we obtain: 
Lemma 3.2 Let $q$ be a odd number. Then, each solution of (1.1) satisfies for $t \in[0, T]$

$$
\begin{aligned}
\int_{\mathcal{U}_{t}^{+}}\left|u_{x}(t)\right|^{q+2} d x+\varepsilon(q+2)(q+1) \int_{0}^{t} \int_{\mathcal{U}_{s}^{+}}\left|u_{x}\right|^{q} u_{x x}^{2} d x d s \\
+\delta(q+2)(q+1) q(n+2) n \int_{0}^{t} \int_{\mathcal{U}_{s}^{+}}\left|u_{x}\right|^{q-1}\left|\mathcal{G}\left(u_{x x}\right)\right| d x d s \\
\quad+(q+1) \int_{0}^{t} \int_{\mathcal{U}_{s}^{+}}\left|u_{x}\right|^{q+3} f^{\prime \prime}(u) d x d s \\
=\int_{\mathcal{U}_{0}^{+}}\left|u_{0}^{\prime}\right|^{q+2} d x,
\end{aligned}
$$

where the last left hand-side term can be replaced by

$$
-(q+2)(q+1) \int_{0}^{t} \int_{\mathcal{U}_{s}+}\left|u_{x}\right|^{q+1} f^{\prime}(u) u_{x x} d x d s .
$$

Now, the combination of Lemmas 3.1 and 3.2 gives the following estimate

Proposition 3.3 Let $\varepsilon, \delta>0$, and $f: \mathbb{R} \rightarrow \mathbb{R}$ be a convex flux function. The solution $u=u_{\varepsilon, \delta}$ of (1.1) - (1.2) satisfies the uniform estimate

$$
\int_{\mathbb{R}}|u(t)|^{\alpha+1} d x+\varepsilon \int_{0}^{t} \int_{\mathbb{R}}|u|^{\alpha-1} u_{x}^{2} d x d s+\delta \int_{0}^{t} \int_{\mathbb{R}}|u|^{\alpha-1}\left|u_{x}\right|\left|u_{x x}\right|^{n} d x d s \leq C_{0},
$$

for all $\frac{5+n}{2 n+1} \leq \alpha<\frac{4+n}{n}$.

Proof. When $g(u)=-|u|^{n}$, equality (3.2) writes

$$
\begin{gathered}
\int_{\mathbb{R}}|u(t)|^{\alpha+1} d x+\alpha(\alpha+1) \varepsilon \int_{0}^{t} \int_{\mathbb{R}}|u|^{\alpha-1} u_{x}^{2} d x d s= \\
\left\|u_{0}\right\|_{\alpha+1}^{\alpha+1}+\alpha(\alpha+1) \delta \int_{0}^{t} \int_{\mathbb{R}}|u|^{\alpha-1} u_{x}\left|u_{x x}\right|^{n} d x d s .
\end{gathered}
$$

Also, when $f$ is convex, we can rewrite (3.7) for $q \geq 1$ odd as

$$
\begin{aligned}
& \int_{\mathcal{U}_{t}^{+}}\left|u_{x}(t)\right|^{q+2} d x+\varepsilon \int_{0}^{t} \int_{\mathcal{U}_{s}^{+}}\left|u_{x}\right|^{q} u_{x x}^{2} d x d s \\
& \quad+\delta \int_{0}^{t} \int_{\mathcal{U}_{s}^{+}}\left|u_{x}\right|^{q-1}\left|u_{x x}\right|^{n+2} d x d s+\int_{0}^{t} \int_{\mathcal{U}_{s}^{+}}\left|u_{x}\right|^{q+3} f^{\prime \prime}(u) d x d s \leq C_{0}
\end{aligned}
$$

However, using the Young inequality, we get

$$
\begin{aligned}
\delta \int_{0}^{t} \int_{\mathcal{U}_{s}^{+}} & |u|^{\alpha-1} u_{x}\left|u_{x x}\right|^{n} d x d s \\
& =\int_{0}^{t} \int_{\mathcal{U}_{s}^{+}}\left(\frac{1}{c t^{\frac{\alpha-1}{\alpha+1}}}|u|^{\alpha-1}\right)\left(c t^{\frac{\alpha-1}{\alpha+1}}\left|u_{x}\right|\right)\left(\delta\left|u_{x x}\right|^{n}\right) d x d s \\
& \leq\left(\frac{1}{t c^{\frac{\alpha+1}{\alpha-1}}}\right)\left(\frac{\alpha-1}{\alpha+1}\right) \int_{0}^{t} \int_{\mathcal{U}_{s}^{+}}|u|^{\alpha+1} d x d s+\frac{c^{k} t^{k \frac{\alpha-1}{\alpha+1}}}{k} \int_{0}^{t} \int_{\mathcal{U}_{s}^{+}}\left|u_{x}\right|^{k} d x d s \\
& +\frac{n}{n+2} \delta^{1+\frac{2}{n}} \int_{0}^{t} \int_{\mathcal{U}_{s}^{+}}\left|u_{x x}\right|^{n+2} d x d s,
\end{aligned}
$$


where $c$ and $k$ are two constants such that

$$
c^{\frac{\alpha+1}{\alpha-1}}=4 \alpha(\alpha-1)
$$

and

$$
\frac{1}{k}+\frac{n}{n+2}+\frac{\alpha-1}{\alpha+1}=1
$$

Thus,

$$
k=\frac{(n+2)(\alpha+1)}{(4+n)-n \alpha}
$$

and if $\frac{5+n}{2 n+1} \leq \alpha<\frac{4+n}{n}$, we get $k \geq 3$.

Now, $q$ is chosen odd such that $2+q \geq k$ to obtain

$$
\left|u_{x}\right|^{k} \leq\left|u_{x}\right|^{3}+\left|u_{x}\right|^{q+2} .
$$

Using (3.11) with $q=1$ and $q \geq k-2$ odd, we obtain

$$
\int_{0}^{t} \int_{\mathcal{U}_{s}^{+}}\left|u_{x}\right|^{k} d x d s+\delta \int_{0}^{t} \int_{\mathcal{U}_{s}^{+}}\left|u_{x x}\right|^{n+2} d x d s \leq C_{0} .
$$

Now, integrating $(3.10)$ over $[0, t]$, we get

$$
\begin{aligned}
& \int_{0}^{t} \int_{\mathcal{U}_{s}^{+}}|u|^{\alpha+1} d x d s \leq \int_{0}^{t} \int_{\mathbb{R}}|u|^{\alpha+1} d x d s \\
& \quad \leq t C_{0}+\alpha(\alpha+1) t \delta \int_{0}^{t} \int_{\mathcal{U}_{s}^{+}}|u|^{\alpha-1} u_{x}\left|u_{x x}\right|^{n} d x d s .
\end{aligned}
$$

Thus, injecting (3.13) and (3.14) in (3.12), it comes

$$
\delta \int_{0}^{t} \int_{\mathcal{U}_{s}^{+}}|u|^{\alpha-1} u_{x}\left|u_{x x}\right|^{n} d x d s \leq C_{0}+\frac{1}{4} \delta \int_{0}^{t} \int_{\mathcal{U}_{s}^{+}}|u|^{\alpha-1} u_{x}\left|u_{x x}\right|^{n} d x d s
$$

and we obtain

$$
\delta \int_{0}^{t} \int_{\mathcal{U}_{s}^{+}}|u|^{\alpha-1} u_{x}\left|u_{x x}\right|^{n} d x d s \leq C_{0} .
$$

Finally, injecting (3.16) in (3.10) we obtain (3.9).

\subsection{Case $f$ convex, $\delta>0$ and $g\left(u_{x x}\right)=-\left|u_{x x}\right|$}

We are concerned here with the equation

$$
u_{t}+f(u)_{x}=\varepsilon u_{x x}-\delta\left|u_{x x}\right|_{x} .
$$

Proposition 3.4 Let $\varepsilon>0$, and $f: \mathbb{R} \rightarrow \mathbb{R}$ be a convex flux function, such that

$$
f^{\prime \prime}(u) \leq C\left(1+|u|^{\beta}\right), \text { where } 0 \leq \beta<3 .
$$

Then, the solution $u=u_{\varepsilon, \delta}$ of (3.17) satisfies the estimate

$$
\int_{\mathbb{R}} u_{x}(t)^{2} d x+\varepsilon \int_{0}^{t} \int_{\mathbb{R}} u_{x x}^{2} d x d s \leq C+\frac{C}{\delta},
$$

where $C>0$ is a constant independent of $\varepsilon$ and $\delta$.

In addition, if $\delta=O\left(\varepsilon^{2}\right)$, the estimate (3.9) with $\alpha=1$ is

$$
\int_{\mathbb{R}} u(t)^{2} d x+\varepsilon \int_{0}^{t} \int_{\mathbb{R}} u_{x}^{2} d x d s+\delta \int_{0}^{t} \int_{\mathbb{R}}\left|u_{x}\right|\left|u_{x x}\right| d x d s \leq C .
$$


Proof. On the one hand, (3.5) is rewritten with $q=0$ as

$$
\begin{array}{rl}
\int_{\mathbb{R}} u_{x}(t)^{2} & d x+2 \varepsilon \int_{0}^{t} \int_{\mathbb{R}} u_{x x}^{2} d x d s \\
=\int_{\mathbb{R}}\left(u_{0}^{\prime}\right)^{2} d x+2 \int_{0}^{t} \int_{\mathbb{R}} f^{\prime}(u) u_{x} u_{x x} d x d s .
\end{array}
$$

Since $f$ satisfies $f^{\prime \prime}(u) \leq C\left(1+|u|^{\beta}\right)$, with $0 \leq \beta<3$. Then

$$
\left|f^{\prime}(u)-f^{\prime}(0)\right| \leq C\left(|u|+|u|^{\beta+1}\right),
$$

where $C$ is a generic constant. Thus,

$$
\begin{aligned}
\left|\int_{0}^{t} \int_{\mathbb{R}} f^{\prime}(u) u_{x} u_{x x} d x d s\right| \leq C & \int_{0}^{t} \int_{\mathbb{R}}|u|\left|u_{x}\right|\left|u_{x x}\right| d x d s \\
& +C \int_{0}^{t} \int_{\mathbb{R}}|u|^{\beta+1}\left|u_{x}\right|\left|u_{x x}\right| d x d s .
\end{aligned}
$$

Applying (3.9) with $n=1, \alpha=2$ and $\alpha=\beta+2<5$, we get

$$
\left|\int_{0}^{t} \int_{\mathbb{R}} f^{\prime}(u) u_{x} u_{x x} d x d s\right| \leq \frac{C}{\delta} .
$$

Finally, injecting (3.22) in (3.20) provides (3.18).

On the other hand, estimate (3.2), with $\alpha=1$ is written as

$$
\int_{\mathbb{R}} u(t)^{2} d x+2 \varepsilon \int_{0}^{t} \int_{\mathbb{R}} u_{x}^{2} d x d s=\left\|u_{0}\right\|_{2}^{2}+2 \delta \int_{0}^{t} \int_{\mathbb{R}} u_{x}\left|u_{x x}\right| d x d s .
$$

Then

$$
\int_{\mathbb{R}} u(t)^{2} d x+2 \varepsilon \int_{0}^{t} \int_{\mathbb{R}} u_{x}^{2} d x d s \leq C_{0}+2 \delta \int_{0}^{t} \int_{\mathbb{R}}\left|u_{x}\right|\left|u_{x x}\right| d x d s .
$$

Now, if $\delta \leq K \varepsilon^{2}$

$$
\begin{aligned}
\delta \int_{0}^{t} \int_{\mathbb{R}}\left|u_{x}\right|\left|u_{x x}\right| d x d s & \leq \frac{\sqrt{\delta}}{\sqrt{K} \varepsilon} \int_{0}^{t} \int_{\mathbb{R}}\left(\sqrt{\varepsilon}\left|u_{x}\right|\right)\left(\sqrt{K \delta \varepsilon}\left|u_{x x}\right|\right) d x d s \\
& \leq \frac{\sqrt{\delta}}{2 \sqrt{K} \varepsilon}\left(\varepsilon \int_{0}^{t} \int_{\mathbb{R}} u_{x}^{2} d x d s+K \delta \varepsilon \int_{0}^{t} \int_{\mathbb{R}} u_{x x}^{2} d x d s\right) \\
& \leq \frac{1}{2}\left(\varepsilon \int_{0}^{t} \int_{\mathbb{R}} u_{x}^{2} d x d s+K \delta \varepsilon \int_{0}^{t} \int_{\mathbb{R}} u_{x x}^{2} d x d s\right) .
\end{aligned}
$$

Using (3.18) and injecting (3.25) in (3.24) give (3.19).

Proposition 3.5 Let $\varepsilon>0, \delta=o\left(\varepsilon^{2}\right)$, and $f: \mathbb{R} \rightarrow \mathbb{R}$ be a convex flux function satisfying

$$
f^{\prime \prime}(u) \leq C\left(1+|u|^{\beta}\right) \text {, where } 0 \leq \beta<3 .
$$

Then, the solution $u=u_{\varepsilon, \delta}$ satisfies
(a) $\left\{\varepsilon u_{x}^{2}\right\}$ is bounded in $L^{1}((0, t) \times \mathbb{R})$.
(b) $\left\{\varepsilon u_{x}\right\} \rightarrow 0$ as $\varepsilon \rightarrow 0$, in $L^{2}((0, t) \times \mathbb{R})$.
(c) $\left\{\delta u_{x}\left|u_{x x}\right|\right\} \rightarrow 0$ as $\varepsilon \rightarrow 0$, in $L^{1}((0, t) \times \mathbb{R})$.
(d) $\left\{\delta\left|u_{x x}\right|\right\} \rightarrow 0$ as $\varepsilon \rightarrow 0$, in $L^{2}((0, t) \times \mathbb{R})$. 
Proof. The statements $(a),(b)$ are obtained thanks to (3.19).

Now, in the same manner we obtained (3.25), we have

$$
\delta \int_{0}^{t} \int_{\mathbb{R}}\left|u_{x}\right|\left|u_{x x}\right| d x d s \leq \frac{\sqrt{\delta}}{2 \varepsilon}\left(\varepsilon \int_{0}^{t} \int_{\mathbb{R}} u_{x}^{2} d x d s+\delta \varepsilon \int_{0}^{t} \int_{\mathbb{R}} u_{x x}^{2} d x d s\right),
$$

and using (3.18) and (3.19) it gets

$$
\delta \int_{0}^{t} \int_{\mathbb{R}}\left|u_{x}\right|\left|u_{x x}\right| d x d s \leq C \frac{\sqrt{\delta}}{\varepsilon}
$$

which gives $(c)$ as soon as $\delta=o\left(\varepsilon^{2}\right)$.

Finally, $(d)$ is obtained thanks to (3.18) since,

$$
\delta^{2} \int_{0}^{t} \int_{\mathbb{R}} u_{x x}^{2} d x d s \leq \frac{\delta}{\varepsilon}\left(\delta \varepsilon \int_{0}^{t} \int_{\mathbb{R}} u_{x x}^{2} d x d s\right) \leq C_{0} \frac{\delta}{\varepsilon} .
$$

\subsection{Case $f$ convex, $\delta>0$ and $g\left(u_{x x}\right)=-u_{x x}^{2}$}

We are concerned here with the equation

$$
u_{t}+f(u)_{x}=\varepsilon u_{x x}-\delta\left(u_{x x}^{2}\right)_{x} .
$$

It is noteworthy that the initial value problem associated with (3.28) is well-posed $[1,2]$.

Proposition 3.6 Let $\varepsilon>0, \delta=O(\varepsilon)$ and $f: \mathbb{R} \rightarrow \mathbb{R}$ be a convex flux function. Then, the solution $u=u_{\varepsilon, \delta}$ of (3.28) satisfies the estimate (3.9) with $\alpha=1$, i.e.,

$$
\int_{\mathbb{R}} u(t)^{2} d x+\varepsilon \int_{0}^{t} \int_{\mathbb{R}} u_{x}^{2} d x d s+\delta \int_{0}^{t} \int_{\mathbb{R}}\left|u_{x}\right| u_{x x}^{2} d x d s \leq C_{0} .
$$

If in addition

$$
f^{\prime \prime}(u) \leq C\left(1+|u|^{\beta}\right), \text { where } 0 \leq \beta<1 / 2,
$$

then the solution $u=u_{\varepsilon, \delta}$ checks

$$
\int_{\mathbb{R}} u_{x}(t)^{2} d x+\varepsilon \int_{0}^{t} \int_{\mathbb{R}} u_{x x}^{2} d x d s \leq C_{0}+C_{0} \delta^{-1 / 2} \varepsilon^{-1 / 4} .
$$

Proof. On the one hand, (3.2) with $n=2, \alpha=1$ is written

$$
\int_{\mathbb{R}} u(t)^{2} d x+2 \varepsilon \int_{0}^{t} \int_{\mathbb{R}} u_{x}^{2} d x d s=\left\|u_{0}\right\|_{2}^{2}+2 \delta \int_{0}^{t} \int_{\mathbb{R}} u_{x} u_{x x}^{2} d x d s,
$$

and, from (3.7) with $q=1$, we get

$$
\varepsilon \int_{0}^{t} \int_{\mathcal{U}_{s}^{+}} u_{x} u_{x x}^{2} d x d s \leq C_{0} .
$$

Thus, if $\delta \leq(k \varepsilon),(3.32)$ and (3.31) give (3.29).

On the other hand, assuming $f^{\prime \prime}(u) \leq C\left(1+|u|^{\beta}\right)$, with $0 \leq \beta<1 / 2$, we have

$$
\left|f^{\prime}(u)-f^{\prime}(0)\right| \leq C\left(|u|+|u|^{\beta+1}\right) .
$$


Then, to estimate the last term in (3.20), we procced as in the case $n=1$ :

$$
\begin{aligned}
\mid \int_{0}^{t} \int_{\mathbb{R}} f^{\prime}(u) u_{x} & \left.u_{x x} d x d s\left|\leq C \int_{0}^{t} \int_{\mathbb{R}}\right| u|| u_{x}|| u_{x x}\left|d x d s+C \int_{0}^{t} \int_{\mathbb{R}}\right| u\right|^{\beta+1}\left|u_{x}\right|\left|u_{x x}\right| d x d s \\
\leq & C \delta^{-1 / 2} \varepsilon^{-1 / 4} \int_{0}^{t} \int_{\mathbb{R}}\left(\delta^{1 / 2}|u|^{1 / 2}\left|u_{x}\right|^{1 / 2}\left|u_{x x}\right|\right)\left(|u|^{1 / 2}\right)\left(\varepsilon^{1 / 4}\left|u_{x}\right|^{1 / 2}\right) d x d s \\
& +C \delta^{-1 / 2} \varepsilon^{-1 / 4} \int_{0}^{t} \int_{\mathbb{R}}\left(\delta^{1 / 2}|u|^{\beta+1 / 2}\left|u_{x}\right|^{1 / 2}\left|u_{x x}\right|\right)\left(|u|^{1 / 2}\right)\left(\varepsilon^{1 / 4}\left|u_{x}\right|^{1 / 2}\right) d x d s
\end{aligned}
$$

Using the Young inequality, it comes

$$
\begin{aligned}
&\left|\int_{0}^{t} \int_{\mathbb{R}} f^{\prime}(u) u_{x} u_{x x} d x d s\right| \leq C \delta^{-1 / 2} \varepsilon^{-1 / 4}\left(\frac{\delta}{2} \int_{0}^{t} \int_{\mathbb{R}}|u|^{2 \beta+1}\left|u_{x}\right| u_{x x}^{2} d x d s\right. \\
&\left.+\frac{\delta}{2} \int_{0}^{t} \int_{\mathbb{R}}|u|\left|u_{x}\right| u_{x x}^{2} d x d s+\frac{1}{2} \int_{0}^{t} \int_{\mathbb{R}} u^{2} d x d s+\frac{\varepsilon}{2} \int_{0}^{t} \int_{\mathbb{R}} u_{x}^{2} d x d s\right) .
\end{aligned}
$$

Inequality (3.9) with $n=2, \alpha=2$ and $\alpha=2 \beta+2<3$ is written as

$$
\delta \int_{0}^{t} \int_{\mathbb{R}}|u|\left|u_{x}\right| u_{x x}^{2} d x d s+\delta \int_{0}^{t} \int_{\mathbb{R}}|u|^{2 \beta+1}\left|u_{x}\right| u_{x x}^{2} d x d s \leq C_{0} .
$$

Now, injecting (3.34) and (3.29) in (3.33), we obtain

$$
\left|\int_{0}^{t} \int_{\mathbb{R}} f^{\prime}(u) u_{x} u_{x x} d x d s\right| \leq C \delta^{-1 / 2} \varepsilon^{-1 / 4}
$$

Finally, injecting (3.35) in (3.20) we get the required estimate (3.30).

Proposition 3.7 Let $\varepsilon>0, \delta=o\left(\varepsilon^{5 / 2}\right)$, and $f: \mathbb{R} \rightarrow \mathbb{R}$ be a convex flux function satisfying

$$
f^{\prime \prime}(u) \leq C\left(1+|u|^{\beta}\right) \text {, where } 0 \leq \beta<1 / 2 .
$$

Then, the solution $u=u_{\varepsilon, \delta}$ of (3.28) satisfies

(a) $\left\{\varepsilon u_{x}^{2}\right\}$ is bounded in $L^{1}((0, t) \times \mathbb{R})$.

(b) $\left\{\varepsilon u_{x}\right\} \rightarrow 0$ as $\varepsilon \rightarrow 0$, in $L^{2}((0, t) \times \mathbb{R})$.

(c) $\left\{\delta u_{x}^{-} u_{x x}^{2}\right\}$, as $u_{x}^{-}=\max \left(0,-u_{x}\right)$, is bounded in $L^{1}((0, t) \times \mathbb{R})$.

(d) $\left\{\delta u_{x}^{+} u_{x x}^{2}\right\} \rightarrow 0$, as $u_{x}^{+}=\max \left(0, u_{x}\right)$ when $\varepsilon \rightarrow 0$ in $L^{1}((0, t) \times \mathbb{R})$.

(e) $\left\{\delta u_{x x}^{2}\right\} \rightarrow 0$ as $\varepsilon \rightarrow 0$, in $L^{1}((0, t) \times \mathbb{R})$.

Proof. The statements $(a),(b)$ and $(c)$ are obtained from (3.29).

Now, $(d)$ is obtained from (3.7) with $q=1$ since

$$
\delta \int_{0}^{t} \int_{\mathcal{U}_{s}+} u_{x} u_{x x}^{2} d x d s \leq \frac{\delta}{\varepsilon}\left(\varepsilon \int_{0}^{t} \int_{\mathcal{U}_{s}+} u_{x} u_{x x}^{2} d x d s\right) \leq C \frac{\delta}{\varepsilon}
$$

Finally, (3.30) provides (e) since

$$
\delta \int_{0}^{t} \int_{\mathbb{R}} u_{x x}^{2} d x d s \leq \delta^{\frac{1}{2}} \varepsilon^{-5 / 4}\left(\delta^{\frac{1}{2}} \varepsilon^{5 / 4} \int_{0}^{t} \int_{\mathbb{R}} u_{x x}^{2} d x d s\right) \leq C \sqrt{\delta \varepsilon^{-5 / 2}} .
$$




\section{Convergence Proof}

We now define the measure-valued solutions to the first order Cauchy problem (1.3)-(1.4) as DiPerna [7].

Definition 4.1 Assume that $u_{0} \in L^{1}(\mathbb{R}) \cap L^{q}(\mathbb{R})$ and $f \in \mathcal{C}(\mathbb{R})$ satisfies the growth condition

$$
|f(u)| \leq \mathcal{O}\left(|u|^{m}\right) \text { as }|u| \rightarrow \infty, \quad \text { for some } m \in[0, q) .
$$

A Young measure $\nu$ is called an entropy measure-valued (e.m.-v.) solution to (1.3)-(1.4) if

$$
\langle\nu,|u-k|\rangle_{t}+\langle\nu, \operatorname{sgn}(u-k)(f(u)-f(k))\rangle_{x} \leq 0, \quad \text { for all } k \in \mathbb{R},
$$

in the sense of distributions on $(0, T) \times \mathbb{R}$, and

$$
\lim _{t \rightarrow 0^{+}} \frac{1}{t} \int_{0}^{t} \int_{K}\left\langle\nu_{(x, s)},\left|u-u_{0}(x)\right|\right\rangle d x d s=0, \quad \text { for all compact set } K \subseteq \mathbb{R} .
$$

A representation theorem of Young's measures associated with a sequence of uniformly bounded functions of $L^{q}$ is used to link the structure of measure and the strong convergence [16].

Lemma 4.2 Let $\left\{u_{n}\right\}_{n \in \mathbb{N}}$ be a bounded sequence in $L^{\infty}\left((0, T) ; L^{q}(\mathbb{R})\right)$. Then there exists a subsequence denoted by $\left\{\tilde{u}_{n}\right\}_{n \in \mathbb{N}}$ and a weakly- $\star$ measurable mapping $\nu: \mathbb{R} \times(0, T) \rightarrow \operatorname{Prob}(\mathbb{R})$ such that, for all functions $h \in \mathcal{C}(\mathbb{R})$ satisfying $(4.1),\left\langle\nu_{(x, t)}, h\right\rangle$ belongs to $L^{\infty}\left((0, T) ; L_{\text {loc }}^{q / m}(\mathbb{R})\right)$ and the following limit representation holds:

$$
\iint_{\mathbb{R} \times(0, T)}\left\langle\nu_{(x, t)}, h\right\rangle \phi(x, t) d x d t=\lim _{n \rightarrow \infty} \iint_{\mathbb{R} \times(0, T)} h\left(\tilde{u}_{n}(x, t)\right) \phi(x, t) d x d t,
$$

for all $\phi \in L^{1}(\mathbb{R} \times(0, T)) \cap L^{\infty}(\mathbb{R} \times(0, T))$.

Conversely, given $\nu$, there exists a sequence $\left\{u_{n}\right\}$ satisfying the same conditions as above and such that (4.4) holds for any $h$ satisfying (4.1).

Proof of the main results. We begin proving (4.2) by using Proposition 3.3, resp. Proposition3.5 , for $n=1$, resp. $n=2$, and we apply the Young measure representation theorem in the suitable $L^{q}$ space (4.4) to show that $\nu$ satisfies (4.2). Also, we use a standard regularization of $\operatorname{sgn}(u-k)(f(u)-f(k))$ and $|u-k|(k \in \mathbb{R})$, since it is sufficient to show that there exists a bounded measure $\mu \leq 0$ such that

$$
\eta(u)_{t}+q(u)_{x} \longrightarrow \mu \quad \text { in } \quad \mathcal{D}^{\prime}(\mathbb{R} \times(0, T))
$$

for an arbitrary convex function $\eta$ (we assume that $\eta^{\prime}$ and $\eta^{\prime \prime}$ are bounded on $\mathbb{R}$ ).

Now, to prove (4.5), we rewrite the formulae (3.1) in the form

$$
\eta(u)_{t}+q(u)_{x}=\mu_{1}+\mu_{2}+\mu_{3}+\mu_{4}
$$

where,

$$
\begin{aligned}
\mu_{1}: & =\varepsilon\left(\eta^{\prime}(u) u_{x}\right)_{x} ; \\
\mu_{2}: & =-\varepsilon \eta^{\prime \prime}(u) u_{x}^{2} ; \\
\mu_{3}: & =\delta\left(\eta^{\prime}(u) g\left(u_{x x}\right)\right)_{x} ; \\
\mu_{4}: & =-\delta \eta^{\prime \prime}(u) u_{x} g\left(u_{x x}\right) .
\end{aligned}
$$

We distinguish the case $n=1$ from $n=2$. 
Case $\mathbf{n}=1: g\left(u_{x x}\right)=-\left|u_{x x}\right|, f$ convex and $\delta=o\left(\varepsilon^{2}\right)$. We have

$$
\begin{gathered}
\left|<\mu_{1}, \theta>\right| \leq \varepsilon \int_{0}^{T} \int_{\mathbb{R}}\left|\theta_{x} \eta^{\prime}(u) u_{x}\right| d x d s \leq \varepsilon \int_{0}^{T} \int_{\mathbb{R}}\left|\theta_{x} u_{x}\right| d x d s \leq C|| \theta_{x}\left\|_{L^{2}}\right\| \varepsilon u_{x} \|_{L^{2}}, \\
\left|<\mu_{2}, \theta>\right| \leq \varepsilon \int_{0}^{T} \int_{\mathbb{R}}\left|\theta \eta^{\prime \prime}(u) u_{x}^{2}\right| d x d s \leq C|| \theta\left\|_{L^{\infty}}\right\| \varepsilon u_{x}^{2} \|_{L^{1}}
\end{gathered}
$$

Since $\eta$ is a convex function, we notice for a non negative function $\theta$

$$
<\mu_{2}, \theta>=-\varepsilon \int_{0}^{T} \int_{\mathbb{R}} \theta \eta^{\prime \prime}(u) u_{x}^{2} d x d s \leq 0 .
$$

In the same way, we have

$$
\begin{aligned}
\left|<\mu_{3}, \theta>\right| & \leq \delta \int_{0}^{T} \int_{\mathbb{R}}\left|\theta_{x} \eta^{\prime}(u)\right| u_{x x}|| d x d s \leq C \delta \int_{0}^{T} \int_{\mathbb{R}}\left|\theta_{x}\right| u_{x x}|| d x d s \\
& \leq C\left\|\theta_{x}\right\|_{L^{2}}|| \delta\left|u_{x x}\right| \|_{L^{2}},
\end{aligned}
$$

and

$$
\begin{aligned}
\left|<\mu_{4}, \theta>\right| & \leq \delta \int_{0}^{T} \int_{\mathbb{R}}\left|\theta \eta^{\prime \prime}(u) u_{x}\right| u_{x x}|| d x d s \leq C \delta \int_{0}^{T} \int_{\mathbb{R}}\left|\theta u_{x}\right| u_{x x}|| d x d s \\
& \leq C\|\theta\|_{L^{\infty}}|| \delta u_{x}\left|u_{x x}\right| \|_{L^{1}} .
\end{aligned}
$$

Combining (4.7), (4.8), (4.9), (4.10) and (4.11), with (a), (b), (c), (d) in Proposition 3.3, gives (4.5) where $\mu$ is non positive bounded measure.

Case $\mathbf{n}=2: g\left(u_{x x}\right)=-u_{x x}^{2}, f$ convex and $\delta=o\left(\varepsilon^{5 / 2}\right)$. Estimates (4.7), (4.8), (4.9) remain true. Concerning $\mu_{3}$, we have

$$
\begin{aligned}
\left|<\mu_{3}, \theta>\right| & \leq \delta \int_{0}^{T} \int_{\mathbb{R}}\left|\theta_{x} \eta^{\prime}(u) u_{x x}^{2}\right| d x d s \leq C \delta \int_{0}^{T} \int_{\mathbb{R}}\left|\theta_{x} u_{x x}^{2}\right| d x d s \\
& \leq C\left\|\theta_{x}\right\|_{L^{\infty}}\left\|\delta u_{x x}^{2}\right\|_{L^{1}} .
\end{aligned}
$$

Now, $\mu_{4}$ is split as

$$
\mu_{4}=\mu_{41}+\mu_{42}
$$

with

$$
\begin{aligned}
& \mu_{41}:=\delta \eta^{\prime \prime}(u) u_{x}^{+} u_{x x}^{2} \\
& \mu_{42}:=-\delta \eta^{\prime \prime}(u) u_{x}^{-} u_{x x}^{2},
\end{aligned}
$$

where $u_{x}^{+}=\max \left(0, u_{x}\right)$ and $u_{x}^{-}=\max \left(0,-u_{x}\right)$. Then we have

$$
\begin{aligned}
\left|<\mu_{41}, \theta>\right| & \leq \delta \int_{0}^{T} \int_{\mathbb{R}}\left|\theta \eta^{\prime \prime}(u) u_{x}^{+} u_{x x}^{2}\right| d x d s \leq C \delta \int_{0}^{T} \int_{\mathbb{R}}\left|\theta u_{x}^{+} u_{x x}^{2}\right| d x d s \\
& \leq C\|\theta\|_{L^{\infty}}\left\|\delta u_{x}^{+} u_{x x}^{2}\right\|_{L^{1}},
\end{aligned}
$$

and

$$
\begin{aligned}
\left|<\mu_{42}, \theta>\right| & \leq \delta \int_{0}^{T} \int_{\mathbb{R}}\left|\theta \eta^{\prime \prime}(u) u_{x}^{-} u_{x x}^{2}\right| d x d s \leq C \delta \int_{0}^{T} \int_{\mathbb{R}}\left|\theta u_{x}^{-} u_{x x}^{2}\right| d x d s \\
& \leq C\|\theta\|_{L^{\infty}}\left\|\delta u_{x}^{-} u_{x x}^{2}\right\|_{L^{1}} .
\end{aligned}
$$


Again, since $\eta^{\prime \prime} \geq 0$, it gets for a non negative function $\theta$

$$
<\mu_{42}, \theta>=-\delta \int_{0}^{T} \int_{\mathbb{R}} \theta \eta^{\prime \prime}(u) u_{x}^{-} u_{x x}^{2} d x d s \leq 0 .
$$

Finally, from inequalities (4.7), (4.8), (4.9), (4.12), (4.13), (4.14) and (4.15), combined with (a), (b), (c) and (d) in Proposition 3.5, we obtain (4.5) where $\mu$ is non positive bounded measure.

Now we will prove (4.3). We follow arguments of DiPerna [7] and Szepessy [18]: we have to check that, for each compact set $K$ of $\mathbb{R}$,

$$
\lim _{t \rightarrow 0+} \frac{1}{t} \int_{0}^{t} \int_{K}\left\langle\nu_{(x, s)},\left|u-u_{0}(x)\right|\right\rangle d x d s=\lim _{t \rightarrow 0+} \lim _{\varepsilon \rightarrow 0+} \frac{1}{t} \int_{0}^{t} \int_{K}\left|u^{\varepsilon, \delta}(x, s)-u_{0}(x)\right| d x d s=0 .
$$

By Jensen's inequality

$$
\frac{1}{t} \int_{0}^{t} \int_{K}\left|u^{\varepsilon, \delta}(x, s)-u_{0}(x)\right| d x d s \leq m(K)^{1 / 2}\left(\frac{1}{t} \int_{0}^{t} \int_{K}\left(u^{\varepsilon, \delta}(x, s)-u_{0}(x)\right)^{2} d x d s\right)^{1 / 2},
$$

where $m(K)$ denotes the Lebesgue measure of $K$. Then we will establish that

$$
\lim _{t \rightarrow 0+\varepsilon \rightarrow 0+} \lim _{t} \frac{1}{t} \int_{0}^{t} \int_{K}\left(u^{\varepsilon, \delta}(x, s)-u_{0}(x)\right)^{2} d x d s=0 .
$$

Let $K_{i} \subset K_{i+1}(i=0,1, \ldots)$ be an increasing sequence of compact sets such that $K_{0}=K$ and $\cup_{i \geq 0} K_{i}=\mathbb{R}$. Using the identity $u^{2}-u_{0}^{2}-2 u_{0}\left(u-u_{0}\right)=\left(u-u_{0}\right)^{2}$, we get for all $i=0,1, \ldots$

$$
\begin{aligned}
& \frac{1}{t} \int_{0}^{t} \int_{K}\left(u^{\varepsilon, \delta}(\cdot, s)-u_{0}\right)^{2} d x d s \\
& \leq \frac{1}{t} \int_{0}^{t}\left(\int_{K_{i}}\left|u^{\varepsilon, \delta}(\cdot, s)\right|^{2} d x-\int_{K_{i}} u_{0}^{2} d x-2 \int_{K_{i}} u_{0}\left(u^{\varepsilon, \delta}(\cdot, s)-u_{0}\right) d x\right) d s \\
& \leq \int_{\mathbb{R} \backslash K_{i}} u_{0}^{2} d x+\frac{1}{t} \int_{0}^{t}\left(\int_{\mathbb{R}}\left|u^{\varepsilon, \delta}(\cdot, s)\right|^{2} d x-\int_{\mathbb{R}} u_{0}^{2} d x\right) \\
& \quad+\frac{2}{t} \int_{0}^{t}\left|\int_{K_{i}} u_{0}\left(u^{\varepsilon, \delta}(\cdot, s)-u_{0}\right) d x\right| d s .
\end{aligned}
$$

For the first term of the right hand side, we clearly have

$$
\lim _{i \rightarrow \infty} \int_{\mathbb{R} \backslash K_{i}} u_{0}^{2} d x=0 .
$$

Now, injecting (3.26) in (3.23), in the case $n=1$, and respectively (3.36) in (3.31), in the case $n=2$, we obtain

$$
\int_{\mathbb{R}}\left|u^{\varepsilon, \delta}(\cdot, s)\right|^{2} d x-\int_{\mathbb{R}} u_{0}^{2} d x \leq C \frac{\sqrt{\delta}}{\varepsilon},
$$

and respectively,

$$
\int_{\mathbb{R}}\left|u^{\varepsilon, \delta}(\cdot, s)\right|^{2} d x-\int_{\mathbb{R}} u_{0}^{2} d x \leq C \frac{\delta}{\varepsilon} .
$$

In both cases, the right hand side of these inequalities tends to zero when $\varepsilon \rightarrow 0$.

To estimate the last term in the inequality (4.16), we choose $\left\{\theta_{n}\right\}_{n \in \mathbb{N}} \subset \mathcal{C}_{0}^{\infty}(\mathbb{R})$ such that

$$
\lim _{n \rightarrow \infty} \theta_{n}=u_{0} \quad \text { in } \quad L^{2}(\mathbb{R}) .
$$


Then, the Cauchy-Schwarz inequality gives

$$
\begin{aligned}
\left|\int_{K_{i}} \quad u_{0}\left(u^{\varepsilon, \delta}(\cdot, s)-u_{0}\right) d x\right| \leq \int_{K_{i}}\left|u_{0}-\theta_{n}\right|\left|u^{\varepsilon, \delta}(\cdot, s)-u_{0}\right| d x \\
+\left|\int_{K_{i}} \theta_{n}\left(u_{0}^{\varepsilon, \delta}-u_{0}\right)+\int_{K_{i}} \theta_{n}\left(u^{\varepsilon, \delta}(\cdot, s)-u_{0}^{\varepsilon, \delta}\right) d x\right| \\
\leq\left\|u_{0}-\theta_{n}\right\|_{L^{2}(\mathbb{R})}\left(\left\|u^{\varepsilon, \delta}(\cdot, s)\right\|_{L^{2}(\mathbb{R})}+\left\|u_{0}\right\|_{L^{2}(\mathbb{R})}\right) \\
+\left\|\theta_{n}\right\|_{L^{2}(\mathbb{R})}\left\|u_{0}^{\varepsilon, \delta}-u_{0}\right\|_{L^{2}(\mathbb{R})}+\left|\int_{0}^{s} \int_{K_{i}} \theta_{n} \partial_{s} u^{\varepsilon, \delta} d x d \tau\right| .
\end{aligned}
$$

In view of (3.19) for $n=1$ and respectively (3.29) for $n=2$, we have

$$
\left\|u_{0}-\theta_{n}\right\|_{L^{2}(\mathbb{R})}\left(\left\|u^{\varepsilon, \delta}(\cdot, s)\right\|_{L^{2}(\mathbb{R})}+\left\|u_{0}\right\|_{L^{2}(\mathbb{R})}\right) \leq\left(\left\|u_{0}\right\|_{L^{2}(\mathbb{R})}+C\right)\left\|u_{0}-\theta_{n}\right\|_{L^{2}(\mathbb{R})},
$$

which tends to zero when $n \rightarrow \infty$, since $\lim _{\varepsilon \rightarrow 0+}\left\|u_{0}^{\varepsilon, \delta}-u_{0}\right\|_{L^{2}(\mathbb{R})}=0$. Finally, it remains to prove that

$$
\lim _{t \rightarrow 0+} \lim _{\varepsilon \rightarrow 0+} \frac{1}{t} \int_{0}^{t}\left|\int_{0}^{s} \int_{K_{i}} \theta_{n} \partial_{s} u^{\varepsilon, \delta} d x d \tau\right| d s=0 .
$$

We have, by (1.1),

$$
\begin{aligned}
\left|\int_{0}^{s} \int_{K_{i}} \theta_{n} \partial_{s} u^{\varepsilon, \delta} d x d \tau\right|= & \left|\int_{0}^{s} \int_{K_{i}} \theta_{n}\left(-f\left(u^{\varepsilon, \delta}\right)_{x}+\varepsilon u_{x x}^{\varepsilon, \delta}+\delta g\left(u_{x x}^{\varepsilon, \delta}\right)_{x}\right) d x d \tau\right| \\
\leq & \int_{0}^{s} \int_{K_{i}}\left|\left(\theta_{n}\right)_{x} f\left(u^{\varepsilon, \delta}\right)\right| d x d \tau+\varepsilon \int_{0}^{s} \int_{K_{i}}\left|\left(\theta_{n}\right)_{x} u_{x}^{\varepsilon, \delta}\right| d x d \tau \\
& +\delta \int_{0}^{s} \int_{K_{i}}\left|\left(\theta_{n}\right)_{x} g\left(u_{x x}^{\varepsilon, \delta}\right)\right| d x d \tau \\
:= & I_{1}+I_{2}+I_{3} .
\end{aligned}
$$

To compute each quantity $I_{1}, I_{2}$ and $I_{3}$, we distinguish the cases $n=1$ from $n=2$.

Case $\mathbf{n}=1: g\left(u_{x x}\right)=-\left|u_{x x}\right|, f$ convex and $\delta=o\left(\varepsilon^{2}\right)$. Since $f$ is such that

$$
0 \leq f^{\prime \prime}(u) \leq C\left(1+|u|^{\beta}\right)
$$

where $\beta<3$. Thus,

$$
|f(u)| \leq C\left(1+|u|^{m}\right),
$$

where $m<5$. Then, Proposition 3.1 implies

$$
\int_{0}^{s} \int_{K_{i}}\left|u^{\varepsilon, \delta}\right|^{m} d x d \tau \leq \int_{0}^{s} \int_{\mathbb{R}}\left|u^{\varepsilon, \delta}\right|^{m} d x d \tau \leq C s
$$

and

$$
\begin{aligned}
I_{1} & =\int_{0}^{s} \int_{K_{i}}\left|\left(\theta_{n}\right)_{x}\right|\left|f\left(u^{\varepsilon, \delta}\right)\right| d x d \tau \\
& \leq C \int_{0}^{s} \int_{K_{i}}\left|\left(\theta_{n}\right)_{x}\right| d x d \tau+C \int_{0}^{s} \int_{K_{i}}\left|\left(\theta_{n}\right)_{x}\right|\left|u^{\varepsilon, \delta}\right|^{m} d x d \tau \\
& \leq C \int_{0}^{s} \int_{K_{i}}\left|\left(\theta_{n}\right)_{x}\right| d x d \tau+C\left\|\left(\theta_{n}\right)_{x}\right\|_{L^{\infty}(\mathbb{R})} \int_{0}^{s} \int_{K_{i}}\left|u^{\varepsilon, \delta}\right|^{m} d x d \tau \\
& \leq C s\left\|\left(\theta_{n}\right)_{x}\right\|_{L^{1}(\mathbb{R})}+C s\left\|\left(\theta_{n}\right)_{x}\right\|_{L^{\infty}(\mathbb{R})} .
\end{aligned}
$$


Thanks to (3.19), we can write

$$
\begin{aligned}
I_{2} & =\varepsilon \int_{0}^{s} \int_{K_{i}}\left|\left(\theta_{n}\right)_{x}\right|\left|u_{x}^{\varepsilon, \delta}\right| d x d \tau \\
& \leq\left(\varepsilon \int_{0}^{s} \int_{K_{i}}\left|\left(\theta_{n}\right)_{x}\right|^{2} d x d \tau\right)^{\frac{1}{2}}\left(\varepsilon \int_{0}^{s} \int_{K_{i}}\left|u_{x}^{\varepsilon, \delta}\right|^{2} d x d \tau\right)^{\frac{1}{2}} \\
& \leq C \varepsilon^{\frac{1}{2}} s^{\frac{1}{2}}\left\|\left(\theta_{n}\right)_{x}\right\|_{L^{2}(\mathbb{R}) .}
\end{aligned}
$$

Finally for $I_{3}$, using (3.27), we get

$$
\begin{aligned}
I_{3} & =\delta \int_{0}^{s} \int_{K_{i}}\left|\left(\theta_{n}\right)_{x} g\left(u_{x x}^{\varepsilon, \delta}\right)\right| d x d \tau=\int_{0}^{s} \int_{K_{i}} \delta\left|\left(\theta_{n}\right)_{x}\right|\left|u_{x x}^{\varepsilon, \delta}\right| d x d \tau \\
& \leq\left(\int_{0}^{s} \int_{K_{i}}\left|\left(\theta_{n}\right)_{x}\right|^{2} d x d \tau\right)^{\frac{1}{2}}\left(\delta^{2} \int_{0}^{s} \int_{K_{i}}\left|u_{x x}^{\varepsilon, \delta}\right|^{2} d x d \tau\right)^{\frac{1}{2}} \\
& \leq s^{\frac{1}{2}}\left\|\left(\theta_{n}\right)_{x}\right\|_{L^{2}(\mathbb{R})}\left(\delta^{2} \int_{0}^{s} \int_{K_{i}}\left|u_{x x}^{\varepsilon, \delta}\right|^{2} d x d \tau\right)^{\frac{1}{2}} \\
& \leq s^{\frac{1}{2}}\left(\frac{\delta}{\varepsilon}\right)^{\frac{1}{2}}\left\|\left(\theta_{n}\right)_{x}\right\|_{L^{2}(\mathbb{R})}
\end{aligned}
$$

Now, from (4.18), (4.19) and (4.20), we deduce

$$
\begin{aligned}
\lim _{t \rightarrow 0+} & \lim _{\varepsilon \rightarrow 0+} \frac{1}{t} \int_{0}^{t}\left|\int_{0}^{s} \int_{K_{i}} \theta_{n} u_{s}^{\varepsilon, \delta} d x d \tau\right| d s \\
\leq & \lim _{t \rightarrow 0+} \lim _{\varepsilon \rightarrow 0+} \frac{C}{t}\left(t^{2}\left(\left\|\left(\theta_{n}\right)_{x}\right\|_{L^{1}(\mathbb{R})}+\left\|\left(\theta_{n}\right)_{x}\right\|_{L^{\infty}(\mathbb{R})}\right)+\varepsilon^{\frac{1}{2}} t^{\frac{3}{2}}\left\|\left(\theta_{n}\right)_{x}\right\|_{L^{2}(\mathbb{R})}\right. \\
& \left.\quad+t^{\frac{3}{2}}\left(\frac{\delta}{\varepsilon}\right)^{\frac{1}{2}}\left\|\left(\theta_{n}\right)_{x}\right\|_{L^{2}(\mathbb{R})} \cdot\right) \\
\leq & \lim _{t \rightarrow 0+\varepsilon \rightarrow 0+} \lim _{\left.\varepsilon \rightarrow \varepsilon^{\frac{1}{2}} t^{\frac{1}{2}}+t^{\frac{1}{2}}\left(\frac{\delta}{\varepsilon}\right)^{\frac{1}{2}}\right)}
\end{aligned}
$$

and since $\delta=o\left(\varepsilon^{2}\right)$, we obtain the desired conclusion, and Theorem 2.1 is proved.

Case $\mathbf{n}=2: g\left(u_{x x}\right)=-u_{x x}^{2}, f$ convex and $\delta=o\left(\varepsilon^{5 / 2}\right)$. Here, $f$ is such that

$$
0 \leq f^{\prime \prime}(u) \leq C\left(1+|u|^{\beta}\right),
$$

where $\beta<1 / 2$, thus,

$$
|f(u)| \leq C\left(1+|u|^{m}\right),
$$

where $m<5 / 2$.

Estimates $I_{1}$ and $I_{2}$ are obtained in the same manner as $n=1$ using (3.29) instead of (3.18). From (3.37) we obtain

$$
\begin{aligned}
I_{3} & =\delta \int_{0}^{s} \int_{K_{i}}\left|\left(\theta_{n}\right)_{x} g\left(u_{x x}^{\varepsilon, \delta}\right)\right| d x d \tau=\int_{0}^{s} \int_{K_{i}} \delta\left|\left(\theta_{n}\right)_{x}\right|\left|u_{x x}^{\varepsilon, \delta}\right|^{2} d x d \tau \\
& \leq\left\|\left(\theta_{n}\right)_{x}\right\|_{L^{\infty}(\mathbb{R})}\left(\delta \int_{0}^{s} \int_{K_{i}}\left|u_{x x}^{\varepsilon, \delta}\right|^{2} d x d \tau\right) \\
& \leq \sqrt{\delta \varepsilon^{-5 / 2}}\left\|\left(\theta_{n}\right)_{x}\right\|_{L^{\infty}(\mathbb{R})}
\end{aligned}
$$


Finally, using (4.18), (4.19) and (4.21), we obtain

$$
\begin{aligned}
& \lim _{t \rightarrow 0+} \lim _{\varepsilon \rightarrow 0+} \frac{1}{t} \int_{0}^{t}\left|\int_{0}^{s} \int_{K_{i}} \theta_{n} u_{s}^{\varepsilon, \delta} d x d \tau\right| d s \\
& \leq \lim _{t \rightarrow 0+} \lim _{\varepsilon \rightarrow 0+} \frac{C}{t}\left(t^{2}\left(\left\|\left(\theta_{n}\right)_{x}\right\|_{L^{1}(\mathbb{R})}+\left\|\left(\theta_{n}\right)_{x}\right\|_{L^{\infty}(\mathbb{R})}\right)+\varepsilon^{\frac{1}{2}} t^{\frac{3}{2}}\left\|\left(\theta_{n}\right)_{x}\right\|_{L^{2}(\mathbb{R})}\right. \\
& \left.\quad+t \sqrt{\delta \varepsilon^{-5 / 2}}\left\|\left(\theta_{n}\right)_{x}\right\|_{L^{\infty}(\mathbb{R})}\right) \\
& \leq \lim _{t \rightarrow 0+} \lim _{\varepsilon \rightarrow 0+} C\left(t+\varepsilon^{\frac{1}{2}} t^{\frac{1}{2}}+\sqrt{\delta \varepsilon^{-5 / 2}}\right)
\end{aligned}
$$

and since $\delta=o\left(\varepsilon^{5 / 2}\right)$, we obtain the desired conclusion, and Theorem 2.2 is proved.

\section{References}

[1] Bedjaoui N., Correia J.M.C., Mammeri Y. Well-posedness of the generalized Korteweg-de Vries-Burgers equation with nonlinear dispersion and nonlinear dissipation. Int. J Pure Math. Vol 2 (2015), 38-46.

[2] Bedjaoui N., Correia J.M.C., Mammeri Y. On vanishing dissipative-dispersive perturbations of hyperbolic conservation laws. Proceedings of the 5th International Conference on Fluid Mechanics and Heat 83 Mass Transfer (2014) Lisbon (Portugal).

[3] Brenier Y., Levy D. Dissipative Behavior of Some Fully Non-Linear KdV-Type Equations. Physica D 137, 2000, pp. 277-294.

[4] Burgers J.M. A Mathematical Model Illustrating the Theory of Turbulence. Adv. Appl. Mech., $1 ; 1948$, p.171.

[5] Correia J.M.C., LeFloch P.G. Nonlinear Diffusive-Dispersive Limits for Multidimensional Conservation Laws, in Nonlinear Partial Differential Equations. World Scientific, Singapore, 1998.

[6] Correia J.M.C., LeFloch P.G. Nonlinear Hyperbolic Conservation Laws, in Nonlinear Evolution Equations and their Applications. Proc. Luso-Chinese Symposium, World Scientific, Singapore, 1999.

[7] DiPerna R.J. Measure-Valued Solutions to Conservation Laws. Arch. Rat. Mech. Anal., 88; 1985, p.223.

[8] Hopf E. The Partial Differential Equation $u_{t}+u u_{x}=\mu u_{x x}$. Comm. Pure Appl. Math., 3; 1950, p.201.

[9] Kružkov S.N. First order quasilinear equations in several independent variables. Mat. Sb., 81; 1970, p.285; Math. USSR Sb., 10; 1970, p.217.

[10] Landau L.D., Lifschitz E.M. Fluid Mechanics. Addison Wesley, Reading, MA, 1971.

[11] Lax P.D., Levermore C.D. The small dispersion limit of the Korteweg-de Vries equation. Comm. Pure Appl. Math., 36; 1983, I, p.253, II, p.571, III, p.809.

[12] LeFloch P.G. Hyperbolic Systems of Conservation Laws. ETH Lect. Notes Ser., BirkhäuserVerlag, Basel, 2002.

[13] P. G. LeFloch and R. Natalini. Conservation laws with vanishing nonlinear diffusion and dispersion, Nonlinear Analysis 36, 1999, pp. 213-230

[14] Marcati P.A., Natalini R. Convergence of the Pseudoviscosity Approximation for Conservation Laws. Nonlin. Anal. T.M.A., 23; 1994, p.621. 
[15] B. Perthame and L. Ryzhik, Moderate Dispersion in Conservation Laws with Convex Fluxes, Comm. Math. Sci. 5(2), 2007, pp. 473-484.

[16] Schonbek M. Convergence of Solutions to Nonlinear Dispersive Equations. Comm. Part. Diff. Equa., 7; 1982, p.959.

[17] M. Slemrod, From Boltzmann to Euler: Hilbert's 6th problem revisited, Computers and Mathematics with Applications 65, 2013, pp. 1497-1501.

[18] Szepessy A. An Existence Result for Scalar Conservation Laws using Measure-Valued Solutions. Comm. Part. Diff. Equa., 14; 1989, p.1329.

[19] Tartar L. The Compensated Compactness Method Applied to Systems of Conservation Laws, in J. Ball, editor, Systems of Nonlinear Partial Differential Equations (Oxford, 1982), vol.111 of NATO ASI Sc. Inst. Ser. C: Math. and Phys. Sc., p.263. D. Reidel publ. Co., Dordrecht-Boston, Mass., 1983.

[20] von Neumann J., Richtmyer R.D. A Method for the Numerical Calculation of Hydrodynamical Shocks. J. Appl. Phys., 21; 1950, p.380.

[21] Whitham G.B. Linear and Nonlinear Waves. Pure \& Appl. Math., Wiley-Interscience Publ., New York, 1974. 